\title{
Structural relaxation of porous glasses due to internal stresses and deformation under tensile loading at constant pressure
}

\author{
Nikolai V. Priezjev ${ }^{1,2}$ and Maxim A. Makeev ${ }^{3}$ \\ ${ }^{1}$ Department of Mechanical and Materials Engineering, \\ Wright State University, Dayton, $\mathrm{OH} 45435$ \\ ${ }^{2}$ National Research University Higher School of Economics, Moscow 101000, Russia and \\ ${ }^{3}$ Department of Chemistry, University of Missouri-Columbia, Columbia, MO 65211
}

(Dated: August 14, 2018)

\begin{abstract}
The time evolution of the pore size distributions and mechanical properties of amorphous solids at constant pressure is studied using molecular dynamics simulations. The porous glasses were initially prepared at constant volume conditions via a rapid thermal quench from the liquid state to the glassy region and allowing for simultaneous phase separation and material solidification. We found that at constant pressure and low temperature, the porous network becomes more compact and the glassy systems relocate to progressively lower levels of the potential energy. Although the elastic modulus and the average glass density both increase with the waiting time, their dependence is described by the power-law function with the same exponent. Moreover, the results of numerical simulations demonstrated that under tensile loading at constant pressure, low-density porous samples become significantly deformed and break up into separate domains at high strain, while dense glasses form a nearly homogeneous solid material.
\end{abstract}

PACS numbers: 34.20.Cf, 68.35.Ct, 81.05.Kf, 83.10.Rs 


\section{INTRODUCTION}

Understanding the influence of pore morphology and porosity on mechanical and physical properties of metallic porous materials is important for structural applications, including wear-resistant tools and biomedical implants, as well as functional applications in catalysis and heat conduction [1, 2, A number of fabrication techniques including phase separation and additive manufacturing open the possibility of obtaining porous structures with various pore sizes, shapes and distributions [1]. Recent experimental and computational studies

have demonstrated that plastic deformation of bulk metallic glasses with a regular array of pores is controlled by shear localization between neighboring pores [3-5]. Moreover, detailed atomistic and continuum simulations have shown that void defects result in shear band nucleation in metallic glasses at strain lower than in uniform samples, and the critical strain depends on the shape and size of the defects [6, 7]. It was also found that tensile plasticity of metallic glass matrix composites can be improved by increasing particle volume fraction, which provides the resistance to shear band propagation [8]. Similarly, the plasticity of metallic glasses under tensile loading can be enhanced by introducing an array of crystalline particles or by increasing temperature [9]. However, the exact relationships between elastic modulus, tensile strength and pore size, shape and distribution have yet to be established.

Using molecular dynamics simulations, it was recently shown that rapid isochoric quenching of a glass-forming mixture from a high temperature liquid state across the glass transition leads to the formation of porous glassy media [10 12]. The kinetics of phase separation and solidification of the glass phase generally depend on the average glass density as well as the rate and depth of thermal quench [10]. In particular, it was demonstrated that after rapid, deep quench, the typical domain size grows as a power-law function of time with the exponent of about 0.5 and then it gradually crosses over to logarithmically slow growth, indicating strongly suppressed kinetics of phase ordering [10]. In the previous study, it was found that the distribution of pore sizes in highly porous systems is well described by a scaling relation up to intermediate length scales, while dense samples with only small isolated pores are characterized by a Gaussian distribution of pore sizes [12]. Moreover, the analysis of local atomic density of solid domains revealed that, with increasing porosity, the local density distribution function develops a broad plateau and a strong peak in the vicinity of zero density, which is characteristic of systems with large surface-to-volume ra- 
tios [12]. Nevertheless, the local density of solid domains away from solid-void interfaces is rather insensitive to porosity and its average value is only slightly below the density of a homogeneous, pore-free glass phase [12].

The mechanical properties and structural transformations of porous glasses at isochoric conditions were recently studied in the cases of shear, tension, and compression using molecular dynamics simulations [13 15]. It was demonstrated that in the linear regime of deformation, the dependence of shear modulus on the average glass density is well described by a power-law function with the exponent of 2.41 [13]. Interestingly, the same exponent was found to hold for the density-dependence of the elastic modulus during tensile and compressive deformations, despite the finite value of normal stress at zero strain that appears in porous samples formed at constant volume [14, 15]. In addition, it was shown that the variation of shear modulus on porosity at low average glass densities is in good agreement with the continuum predictions based on the percolation theory [13, 16]. Upon further increasing strain, the pore shapes become significantly deformed and adjacent pores coalesce with each other, leading to formation of large voids, necking and eventually breaking of the material [13, 14]. At high compressive strain, the pore coalescence and void redistribution result in the formation of nearly homogeneous solid domains, which provide enhanced resistance to deformation [15]. However, the influence of various deformation protocols (e.g., constant pressure vs. constant volume deformation and stress vs. strain-controlled loading) on mechanical and structural properties of porous glasses remains not fully understood.

In this paper, the structural relaxation of porous glasses that are initially prepared at constant volume, is studied in the NPT ensemble using molecular dynamics simulations. It will be shown that porous samples become significantly compressed under built-in tensile stresses, which is reflected in the time evolution of the average glass density and the shape of the pore size distribution functions. The slow aging process is also characterized by the gradual decrease in the potential energy. The mechanical properties are probed at different waiting times by imposing tensile strain at constant pressure. It is found that the functional form of the elastic modulus as a function of the average glass density holds for tensile loading at constant volume as well as at constant pressure and different waiting times. The analysis of the atomic configurations and pore size distributions helps to distinguish the process of breaking of the porous samples at large strain for lower average glass densities from the 
formation of a nearly homogeneous, high-density amorphous material.

The paper is organized as follows. The details of molecular dynamics simulations as well as the preparation and deformation protocols are described in the next section. The analysis of the pore size distributions, potential energy, density profiles, and mechanical properties of porous glasses are presented in Sec.III. The brief summary is given in the last section.

\section{MD SIMULATION DETAILS}

The molecular dynamics simulations described below were performed on a model glass former represented by the Kob-Andersen (KA) binary mixture [17]. Our system consists of 240000 large atoms of type $A$ and 60000 small atoms of type $B$, thus making the total number of atoms $N=300000$. Upon cooling, the crystallization of the KA mixture is suppressed because of the non-additive Lennard-Jones (LJ) interactions [17]. More specifically, the interaction between any two atoms of types $\alpha, \beta=A, B$ is defined by the LJ potential:

$$
V_{\alpha \beta}(r)=4 \varepsilon_{\alpha \beta}\left[\left(\frac{\sigma_{\alpha \beta}}{r}\right)^{12}-\left(\frac{\sigma_{\alpha \beta}}{r}\right)^{6}\right]
$$

with the following parametrization $\varepsilon_{A A}=1.0, \varepsilon_{A B}=1.5, \varepsilon_{B B}=0.5, \sigma_{A B}=0.8$, and $\sigma_{B B}=0.88$ [17]. The mass of each atom type is the same, $m_{A}=m_{B}$. To speed up computation, the LJ potential is truncated at the cutoff radius $r_{c, \alpha \beta}=2.5 \sigma_{\alpha \beta}$. Throughout the paper, the results are reported in the reduced units of length, mass, energy, and time, i.e., $\sigma=\sigma_{A A}, m=m_{A}, \varepsilon=\varepsilon_{A A}$, and $\tau=\sigma \sqrt{m / \varepsilon}$, respectively. The numerical integration of the Newton's equations of motion was carried out using the velocity-Verlet algorithm [18, 19] with the time step $\triangle t_{M D}=0.005 \tau$.

The initial equilibration of the system in the periodic box of constant volume and the subsequent thermal quench across the glass transition is identical to the preparation procedure implemented in the previous MD studies [10 15]. Here, we briefly describe the numerical protocol, which results in the formation of the porous glass. The system was initially equilibrated at constant volume and the temperature of $1.5 \varepsilon / k_{B}$ during $3 \times 10^{4} \tau$. The Boltzmann constant is denoted by $k_{B}$. This temperature is well above the glass transition temperature of the KA model $T_{g} \approx 0.435 \varepsilon / k_{B}$ at the atomic density $\rho=\rho_{A}+\rho_{B}=1.2 \sigma^{-3}$ [17]. Next, the temperature was instantaneously reduced to the target value $T=0.05 \varepsilon / k_{B}$, and the system was allowed to evolve freely at constant volume during the time interval of $10^{4} \tau$. At the low 
temperature, the phase separation kinetics leads to the formation of porous structure in the amorphous solid [10 15]. In the present study, the simulation results were carried out only for one realization of disorder due to computational limitations.

After the porous glass samples were prepared at constant volume, the follow-up MD simulations were performed in the isothermal-isobaric $(N P T)$ ensemble, where $P=0$ and $T=0.05 \varepsilon / k_{B}$. The temperature $T=0.05 \varepsilon / k_{B}$ was regulated via the Nosé-Hoover thermostat [18]. As discussed in detail in the next section, the relaxation process at constant pressure results in the gradual increase of the average glass density even in the absence of external deformation. The periodic boundary conditions were applied in all three dimensions, which were allowed to change independently during densification of the porous samples. Next, the tensile deformation was imposed along the $\hat{x}$ direction with the strain rate $\dot{\varepsilon}_{x x}=10^{-4} \tau^{-1}$, while the pressure in the $\hat{y}$ and $\hat{z}$ directions was maintained at zero. The maximum strain was varied from $100 \%$ to $400 \%$ depending on the average glass density. During the production runs, the pressure components, potential energy, system dimensions as well as atomic configurations were saved for the postprocessing analysis of the porous structure and mechanical properties.

\section{RESULTS}

As discussed in the previous section, the formation of porous glassy systems occurs in the process of concurrent phase separation and material solidification after sufficiently deep thermal quench from a liquid state [10, 11]. The kinetics of the process at low temperature and constant volume becomes logarithmically slow and the particle diffusion in the glass phase is strongly suppressed [10, 11]. An example of the porous structure in the amorphous solid formed at constant volume after the long time interval of $10^{4} \tau$ is shown in Fig. 1 (a) for the average glass density $\rho \sigma^{3}=0.3$. Notice the complex interconnected pore network embedded into a dense glass phase. Thus formed porous samples in a wide range of average glass densities, $0.2 \leqslant \rho \sigma^{3} \leqslant 1.0$, were used as starting configurations for the MD simulations performed in the NPT ensemble and described in the present study.

It should be particularly emphasized that the porous glass formation at constant volume and low temperature occurs at a negative pressure, which depends on the target temperature and the average glass density [12]. Moreover, it was recently deduced from extensive 
molecular dynamics simulations that the data in a wide range of parameters can be collapsed onto a scaling relation of the form $P / T \sim \rho^{\alpha}$ with the exponent $\alpha \approx 2.5$ [12]. Thus, at the temperature $T=0.05 \varepsilon / k_{B}$ considered in the present study, the average values of pressure vary from $P \approx-0.73 \varepsilon / \sigma^{3}$ for $\rho \sigma^{3}=0.9$ to $P \approx-0.01 \varepsilon / \sigma^{3}$ for $\rho \sigma^{3}=0.2$. In other words, the glassy porous systems can be viewed as being under tension in a confined geometry. It is, therefore, expected that when the constraint of constant volume is relaxed and the simulations are instead performed at constant pressure, the porous samples will undergo isotropic compression, which is driven by the build-in tensile stresses. The evolution of atomic configurations at zero pressure and temperature $T=0.05 \varepsilon / k_{B}$ is shown in Fig. 1 for the indicated waiting times. It can be clearly observed that the solid phase and the porous network become gradually compressed and the average glass density increases over time.

A more quantitative analysis of the relaxation process under internal stresses at constant pressure, $P=0$, can be performed by considering the time dependence of the average glass density and the potential energy. First, the variation of the average glass density as a function of the waiting time is plotted in Fig.2. It can be seen that the most rapid densification occurs after the time interval of about $100 \tau$, which is followed by a slow aging process up to $2.5 \times 10^{5} \tau$. As evident, the relative increase in the average glass density during the time interval of $2.5 \times 10^{5} \tau$ is more pronounced in lower density samples. Note also that the average glass density of the two dense samples (two upper curves in Fig. 2) becomes nearly the same after about $10^{4} \tau$. We comment that these dense samples are not pore-free even after the time interval of $2.5 \times 10^{5} \tau$ as their average density is still below the density of the homogeneous glass phase of about $1.21 \sigma^{-3}$ at zero pressure [25]. Second, the time dependence of the potential energy per atom for the same porous samples is reported in Fig. 3. Similar to the case of density, the potential energy starts to decrease rapidly after about $100 \tau$ followed by a gradual decay during the next two decades, which suggests that the relaxation dynamics becomes progressively more slow. The lowest potential energy is also attained in two dense samples at $t \gtrsim 10^{4} \tau$.

The analysis of pore structure and porosity evolution in the systems under consideration is based on calculation of the pore size distribution (PSD) functions. To this end, the opensource Zeo++ software was utilized [20 22]. The pore sizes were computed using algorithms implemented in Zeo++ [20 22]. The approach involves the Voronoi decomposition of the 
total volume of system into Voronoi cells, associated with each individual atom in the system. The implementation derives from a modification to the $\mathrm{VORO}++$ software library, developed in Ref. [23]. This computational tool provides Voronoi network and all the parameters necessary for analysis of the geometrical characteristics of void space in porous material systems. The Voronoi decomposition is performed such that the space surrounding atoms is divided into polyhedral cells and each face of the polyhedral is a plane equidistant from the two points sharing the face. Edges of the cells correspond to lines equidistant to neighboring points, whereas vertices are equidistant from neighboring points. The Voronoi network, built thereby, maps the void space in porous systems. Further, the computed Voronoi network can be utilized to obtain quantitative information on the largest pore in the systems and the largest pore that can traverse through it, topological properties of pores and channels, and other geometric quantities. It should be noted that Zeo ++ allows one to obtain information for model systems with periodic structural units, comprised of multiple types of atoms. For a given radius of the probe, one can identify the probe accessible regions of the voidspace network using a graph propagation algorithm. In practice, a variation of the Dijkstra shortest path algorithm is used [24]. In this work, the analysis was used to identify the topological properties of channels in the porous systems. The surface areas of the pores and their volumes are computed using a Monte Carlo sampling. The number of samples per atom used in this works is fixed at 50000. The probe radius used for calculations is $0.3 \sigma$. The value of the probe radius was varied in a wide range and no significant variations in the results were observed for values less than $1.0 \sigma$.

The pore size distribution functions during the relaxation process at constant pressure are presented in Fig.4 for the indicated waiting times. These data were computed in three porous samples after the constant pressure condition was applied in the absence of external loading. It can be clearly observed that with increasing average glass density, the pore size distributions at $t=0$ become more narrow. This behavior is consistent with the results of the previous MD studies, where simulations were performed at constant volume [12 15]. The distribution functions presented in Fig.4(a) correspond to atomic configurations shown in Fig. 1 as well as blue curves in Figs. 2 and 3 . It can be seen that, as the pore network becomes more compact, the shape of distributions are skewed toward smaller length scales. In agreement with the slow variation of the average glass density and potential energy at large waiting times, the PSDs at $t=5 \times 10^{4} \tau$ and $2.5 \times 10^{5} \tau$ are nearly the same, apart from 
statistical fluctuations. The case of higher density porous glass shown in Fig. 4(b) appears to be qualitatively similar, although the average pore size is smaller. In contrast, the pore size distributions in the case shown in Fig. 4. (c) evolve to the symmetric, bell-shaped curve at large times, which is representative of low-porosity systems with a collection of isolated pores [12].

We next discuss the mechanical response of the porous glass to tensile loading at constant pressure $P=0$ and temperature $T=0.05 \varepsilon / k_{B}$. In Fig. 5 , the stress-strain curves are plotted for nine samples with different average glass densities after the waiting time of $5 \times 10^{4} \tau$. In can be seen that both the slope of the linear region and the yield stress become larger with increasing average glass density. We comment that in contrast to a finite stress at zero strain in porous glasses prepared and strained at constant volume [14, 15], the stress is initially zero in all samples shown in Fig.5. Furthermore, at larger strain, $\varepsilon_{x x} \gtrsim 0.1$ in Fig. 5, the stress curves exhibit a shallow maximum and then decay to zero, indicating breakup of the amorphous material into separate domains (discussed below). Notice that the failure of denser samples occurs at higher values of strain. However, in the three cases of higher density samples, the stress appears to saturate to a broad plateau, which extends up to $\varepsilon_{x x}=2.0$. The stress amplitude at the plateau level is determined by the deformation of a nearly homogeneous glass phase, where almost all pores got transformed and ejected from the amorphous material.

The elastic modulus, $E$, computed in the linear regime of deformation after the waiting time of $5 \times 10^{4} \tau$ is shown in the inset to Fig. 5 as a function of the average glass density. As is evident, the elastic modulus follows a power-law dependence $E \sim \rho^{2.41}$, which is indicated by the straight dashed line. Interestingly, the same exponent was reported in our previous studies of tension, compression, and shear of porous glasses at constant volume [13 15]. For reference, the data for tension of porous glasses at constant volume [14] are also plotted in the inset to Fig.5. As shown in Fig.2, the average glass density in all samples gradually increases over time even in the absence of external deformation, and, therefore, it is expected that the elastic modulus will be larger for more aged samples. Indeed, the data collected after longer waiting time of $15 \times 10^{4} \tau$ reveal that although the elastic modulus and the average glass density slightly increase in each sample, the data still follow the same powerlaw dependence as for the less aged glasses (see inset in Fig.5). These results demonstrate 
that the functional form of the density-dependent elastic modulus of porous glasses is the same for both deformation protocols, i.e., tension at constant volume and constant pressure.

The representative snapshots of strained porous glasses after the waiting time of $5 \times 10^{4} \tau$ are presented in Figs.6, 7, and 8 for different average glass densities. The atomic configurations for three samples are shown for the indicated values of strain along the stress-strain curves reported in Fig.5. It can be clearly seen that the pore configurations in less dense samples (shown in Figs.6 and 7) become significantly distorted, leading to necking and eventually breaking of the material at large strain. These results are qualitatively similar to the cases of tension at constant volume reported in the previous MD study [14]. Note, however, that the average glass density increases slightly during tension at constant pressure, except at large strain in Figs.6(d) and 7(d) where the formation of system-spanning voids contributes to the apparent decrease in the average glass density. By contrast, the deformation of the dense porous sample proceeds via the formation of increasingly dense and homogeneous glass phase (see Fig. 8). Notice that some isolated pores become significantly elongated in highly strained samples. We also checked that necking is not formed in the dense porous glass during tension at constant pressure up to $\varepsilon_{x x} \leqslant 4.0$ (not shown).

The evolution of pore size distribution functions during tensile loading after the waiting time of $5 \times 10^{4} \tau$ are shown in Fig.9 for the indicated values of strain. Note that the distributions plotted in the panels (a), (b), and (c) in Fig.9 were computed using atomic configurations presented in Figs.6, 7, and 8, respectively. It can be seen in Fig.9.9(a-b) that lower density porous samples show the same trend; namely, the pore size distributions are initially narrow and peaked at $10 \lesssim d_{p} / \sigma \lesssim 15$. With increasing strain, the PSDs become more broad and a distinct peak develops at large length scales, which corresponds to the formation of a large void associated with material failure (see Figs.6 and 7). These results are similar to the breaking process during tensile loading at constant volume reported in our previous study [14]. By sharp contrast, the pore size distributions for the higher density sample in Fig.9(c) remain confined to small length scales and the amplitude of PSDs becomes significantly reduced at high strain. This behavior correlates well with visual observation of strained samples in Fig.8, where only a few isolated, highly deformed pores remain at high strain, thus forming a nearly homogeneous glassy material.

As shown above, a significant rearrangement of the material takes place during the tran- 
sition to zero-pressure state as well as in the process of mechanical loading. Therefore, it is important to quantify the temporal evolution of density variations in the systems. In our previous studies [13-15], we analyzed temporal evolution of the density profiles in model porous glasses undergoing mechanical loading at constant volume. In what follows, we apply a similar analysis to the atomic systems under consideration. The methodology of analysis has been previously described in detail in Refs. [13 15]. A brief summary is as follows. We numerically compute spatially-resolved density profiles along the direction of externally applied strain. The quantity of interest is denoted by $\langle\rho\rangle_{s}(x)$ and defined as the number of atoms of either type, located in a bin with thickness $b \approx \sigma$ along the loading direction, and divided by the volume of the bin, $b L_{y} L_{z}$, where $L_{y}$ and $L_{z}$ are the box sizes in the two Cartesian directions perpendicular to the loading direction.

Similar to the previous cases of tensile [14] and compressive [15] loadings at constant volume, the analysis of spatially-resolved density profiles reveals a number of notable features pertained to density rearrangement during tension at constant pressure. In particular, differences observed in systems with low and high average densities are worth noting. The simulation results are reported for three samples in Figs. 10, 11, and 12, At relatively low glass densities, shown in Figs. 10 and 11, the mechanical failure occurs in the regions with largest spatial extent of local density deviations from its average value. As can be observed in Figs. 10 and 11, a dip in $\langle\rho\rangle_{s}(x)$ starts to develop within the regions with low average densities. The process of local density decrease in these regions is accompanied by simultaneous densification in the neighboring parts of the system, similar to the constant volume loading conditions [14]. Also, the shapes of density patterns are largely preserved in the parts of the systems outside of the low-density region of largest extent. Specifically, the density patterns repeat themselves with increasing strain, the only difference being their magnitudes and lateral shifts. The observed increase in density magnitudes is due to rearrangement of material when it flows from the low-density region to the regions of elevated densities. This type of behavior has also been observed in porous glasses loaded at constant volume [14].

In contrast, at higher average glass densities, shown in Fig.12, the tensile loading at constant pressure leads to qualitatively different evolution of the density profiles. In this case, the deformation results in gradual closure of the existing pores. Specifically, the density profiles reveal a gradual increase in density throughout the sample in the range of intermediate 
strains. At large strains, the density profiles become nearly flat outside of the region with largest extent of deviation from the average density. Note, however, a small dip of about $10 \%$ remains at strain $\varepsilon_{x x}=1.6$. This behavior differs from the one observed for loading at constant volume, where the dip in the large-extent low-density region develops with increasing strain [14]. These differences are due to a competition between two effects. One of the effects is the material rearrangement within the system and the other is the volume change due to relaxation at constant pressure. The latter leads to a decrease in total volume, which does not occur in systems undergoing evolution at fixed volume.

\section{CONCLUSIONS}

In summary, dynamical evolution of porous glasses from a constant volume state to zero-pressure state and mechanical response properties of glasses at constant pressure were investigated using atomistic simulations. We found that the transition to the zero-pressure state is accompanied by significant increase in average density and corresponds to a transition to lower energy states. The temporal evolution of the pore distribution functions during structural relaxation at zero pressure were examined. It was revealed that a transition to configurations with a narrower pore-size distributions is energetically favorable as compared to the larger pores supported by built-in tensile stresses in the systems. Both constantvolume and constant pressure configurations, in the absence of deformation, show randomly distributed, isolated ensembles of pores at higher average glass densities, while interconnected porous structure is formed at lower densities. At constant pressure, the equilibrium configurations consist of narrower distributions of pores, which preserve, however, the major characteristics of pore-size distribution functions observed for samples equilibrated at constant volume.

The mechanical response properties, studied at zero pressure, reveal a number of noteworthy features. First, we found the exponent in the power-law scaling of the elastic modulus with density is the same as in the case of tensile, compressive, and shear testing of the systems at constant volume. Consequently, the effect of built-in pressures on elastic properties manifests itself primarily in the corresponding porosity changes. Moreover, as our studies show, the mechanical loading of porous systems at constant pressure differs from that at fixed volume by the effect of total volume change. The evolutions of pore ensembles in the 
systems subjected to tension, is similar to the ones characteristic for the constant-volume loadings at smaller average densities. In these cases, pore growth and coalescence is favored as compared to the changes in the system volume. At higher densities, however, the total volume changes become the dominant effect in the response to the external loading. This manifests itself in distinctly different behavior of density profiles in the regime of higher densities as compared to the less dense samples.

\section{Acknowledgments}

Financial support from the National Science Foundation (CNS-1531923) is gratefully acknowledged. The article was prepared within the framework of the Basic Research Program at the National Research University Higher School of Economics (HSE) and supported within the framework of a subsidy by the Russian Academic Excellence Project '5-100'. The molecular dynamics simulations were performed using the LAMMPS numerical code [18]. The distributions of pore sizes were computed using the open-source software ZEO++ developed at the Lawrence Berkeley National Laboratory [20 22]. Computational work in support of this research was performed at Wright State University's High Performance Computing Facility and the Ohio Supercomputer Center.

[1] B. Zhao, A. K. Gain, W. Ding, L. Zhang, X. Li, and Y. Fu, A review on metallic porous materials: pore formation, mechanical properties, and their applications, Int. J. Adv. Manuf. Technol. 95, 2641 (2018).

[2] X. Wang, S. Xu, S. Zhou, W. Xu, M. Leary, P. Choong, M. Qian, M. Brandt, Y. M. Xie, Topological design and additive manufacturing of porous metals for bone scaffolds and orthopaedic implants: A review, Biomaterials 83, 127 (2016).

[3] B. Sarac, B. Klusemann, T. Xiao, and S. Bargmann, Materials by design: An experimental and computational investigation on the microanatomy arrangement of porous metallic glasses, Acta Mater. 77, 411 (2014).

[4] B. Sarac, D. Sopu, E. Park, J. K. Hufenbach, S. Oswald, M. Stoica, and J. Eckert, Mechanical and structural investigation of porous bulk metallic glasses, Metals 5, 920 (2015). 
[5] D. Sopu, C. Soyarslan, B. Sarac, S. Bargmann, M. Stoica, and J. Eckert, Structure-property relationships in nanoporous metallic glasses, Acta Mater. 106, 199 (2016).

[6] S. Gouripriya and P. Tandaiya, Mechanisms of compressive deformation and failure of porous bulk metallic glasses, Modelling Simul. Mater. Sci. Eng. 25, 045006 (2017).

[7] Y. Luo, G. Yang, Y. Shao, and K. Yao, The effect of void defects on the shear band nucleation of metallic glasses, Intermetallics 94, 114 (2018).

[8] Y. Jiang, L. Sun, Q. Wu, and K. Qiu, Enhanced tensile ductility of metallic glass matrix composites with novel microstructure, J. Non-Cryst. Solids 459, 26 (2017).

[9] H. Y. Song, S. Li, Y. G. Zhang, Q. Deng, T. H. Xu, and Y. L. Li, Atomic simulations of plastic deformation behavior of $\mathrm{Cu}_{50} \mathrm{Zr}_{50}$ metallic glass, J. Non-Cryst. Solids 471, 312 (2017).

[10] V. Testard, L. Berthier, and W. Kob, Influence of the glass transition on the liquid-gas spinodal decomposition, Phys. Rev. Lett. 106, 125702 (2011).

[11] V. Testard, L. Berthier, and W. Kob, Intermittent dynamics and logarithmic domain growth during the spinodal decomposition of a glass-forming liquid, J. Chem. Phys. 140, 164502 (2014).

[12] M. A. Makeev and N. V. Priezjev, Distributions of pore sizes and atomic densities in binary mixtures revealed by molecular dynamics simulations, Phys. Rev. E 97, 023002 (2018).

[13] N. V. Priezjev and M. A. Makeev, Evolution of the pore size distribution in sheared binary glasses, Phys. Rev. E 96, 053004 (2017).

[14] N. V. Priezjev and M. A. Makeev, Strain-induced deformation of the porous structure in binary glasses under tensile loading, Comput. Mater. Sci. 150, 134 (2018).

[15] N. V. Priezjev and M. A. Makeev, Structural transformations and mechanical properties of porous glasses under compressive loading, J. Non-Cryst. Solids (2018). In press. DOI: https://doi.org/10.1016/j.jnoncrysol.2018.04.008

[16] M. Sahimi, Applications of Percolation Theory (Taylor and Francis, London, 1994).

[17] W. Kob and H. C. Andersen, Testing mode-coupling theory for a supercooled binary LennardJones mixture: The van Hove correlation function, Phys. Rev. E 51, 4626 (1995).

[18] S. J. Plimpton, Fast parallel algorithms for short-range molecular dynamics, J. Comp. Phys. 117, 1 (1995).

[19] M. P. Allen and D. J. Tildesley, Computer Simulation of Liquids (Clarendon, Oxford, 1987).

[20] R. L. Martin, B. Smit, and M. Haranczyk, Addressing challenges of identifying geometrically 
diverse sets of crystalline porous materials, J. Chem. Inf. Model. 52, 308 (2012).

[21] T. F. Willems, C. H. Rycroft, M. Kazi, J. C. Meza, and M. Haranczyk, Algorithms and tools for high-throughput geometry-based analysis of crystalline porous materials, Micropor. Mesopor. Mater. 149, 134 (2012).

[22] D. Ongari, P. G. Boyd, S. Barthel, M. Witman, M. Haranczyk, and B. Smit, Accurate characterization of the pore volume in microporous crystalline materials, Langmuir 33, 14529 (2017).

[23] C. H. Rycroft, VORO++: A three-dimensional Voronoi cell library in C++, Chaos 19, 041111 (2009).

[24] E. W. Dijkstra, A note on two problems in connexion with graphs, Numerische Mathematik 1, 269 (1959).

[25] N. V. Priezjev, Slow relaxation dynamics in binary glasses during stress-controlled, tensioncompression cyclic loading, Comput. Mater. Sci. 153, 235 (2018). 
Figures 
(a)

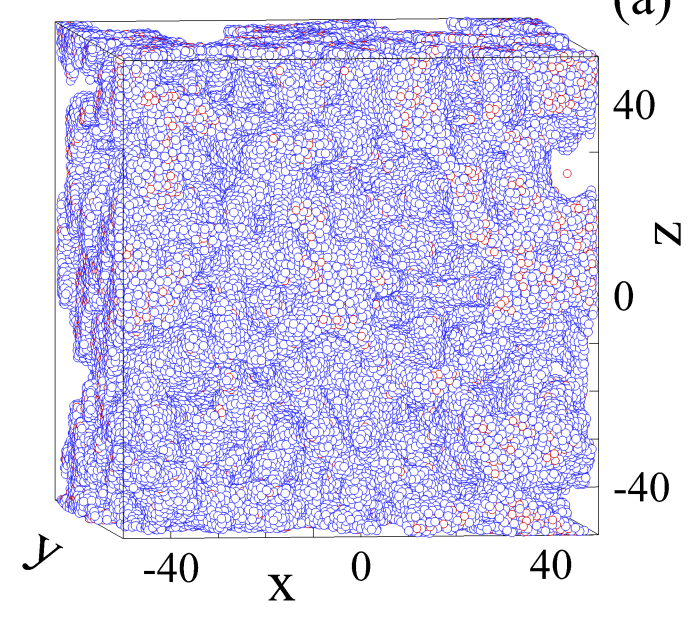

(c)

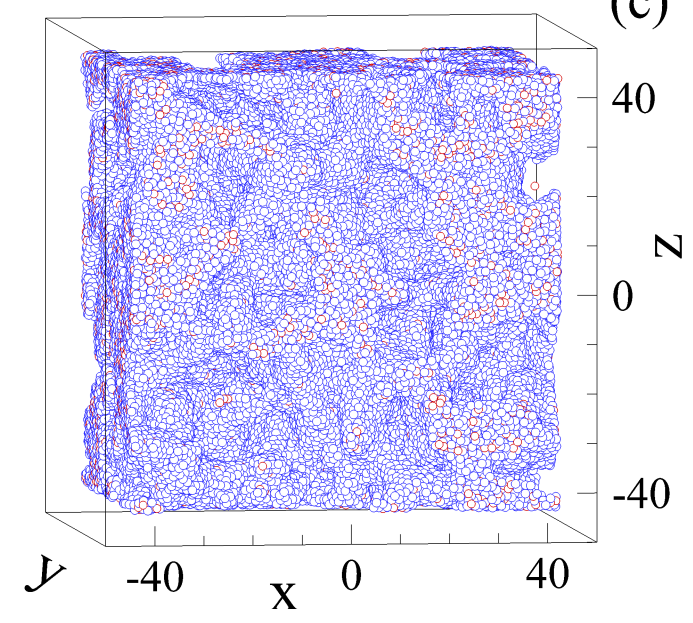

(b)

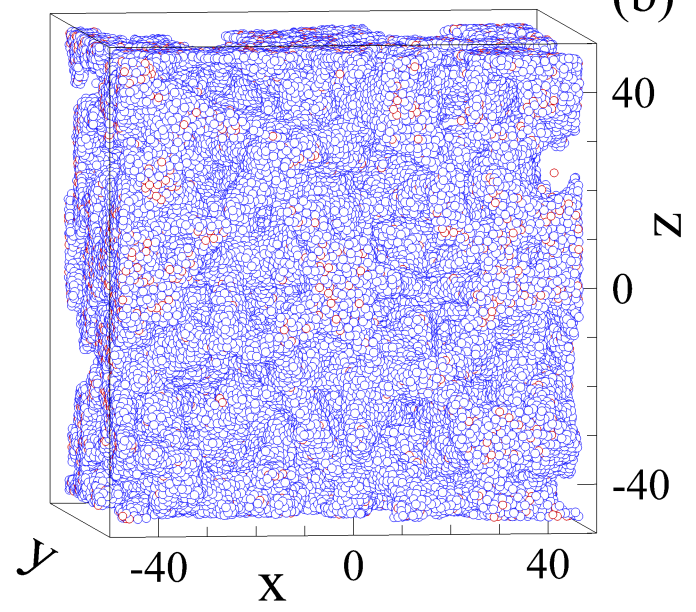

(d)

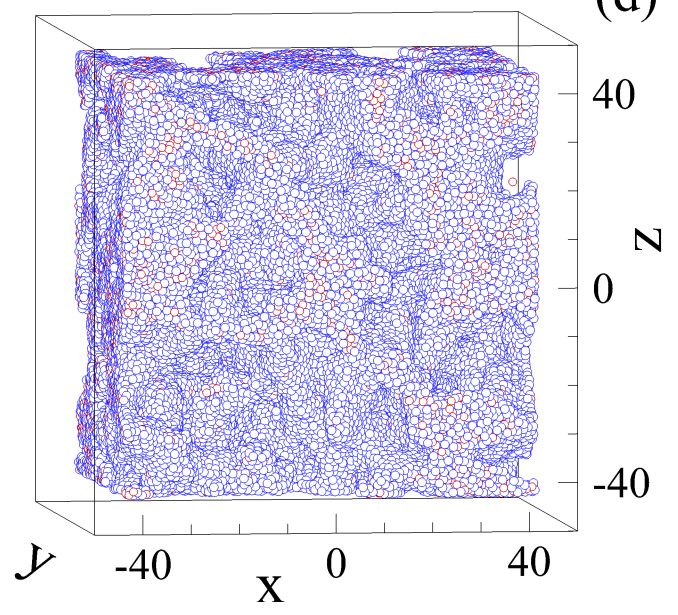

FIG. 1: (Color online) A series of snapshots of the porous glass at zero pressure after time intervals (a) $0 \tau$, (b) $500 \tau$, (c) $50000 \tau$, and (d) $250000 \tau$. The average glass densities are (a) $\rho \sigma^{3}=0.30$, (b) $\rho \sigma^{3}=0.37$, (c) $\rho \sigma^{3}=0.49$, and (d) $\rho \sigma^{3}=0.52$. The temperature is $T=0.05 \varepsilon / k_{B}$ and the total number of atoms is $N=300000$. Atoms of types $A$ and $B$ are denoted by blue and red spheres. Note that the size of atoms is reduced for clarity. 


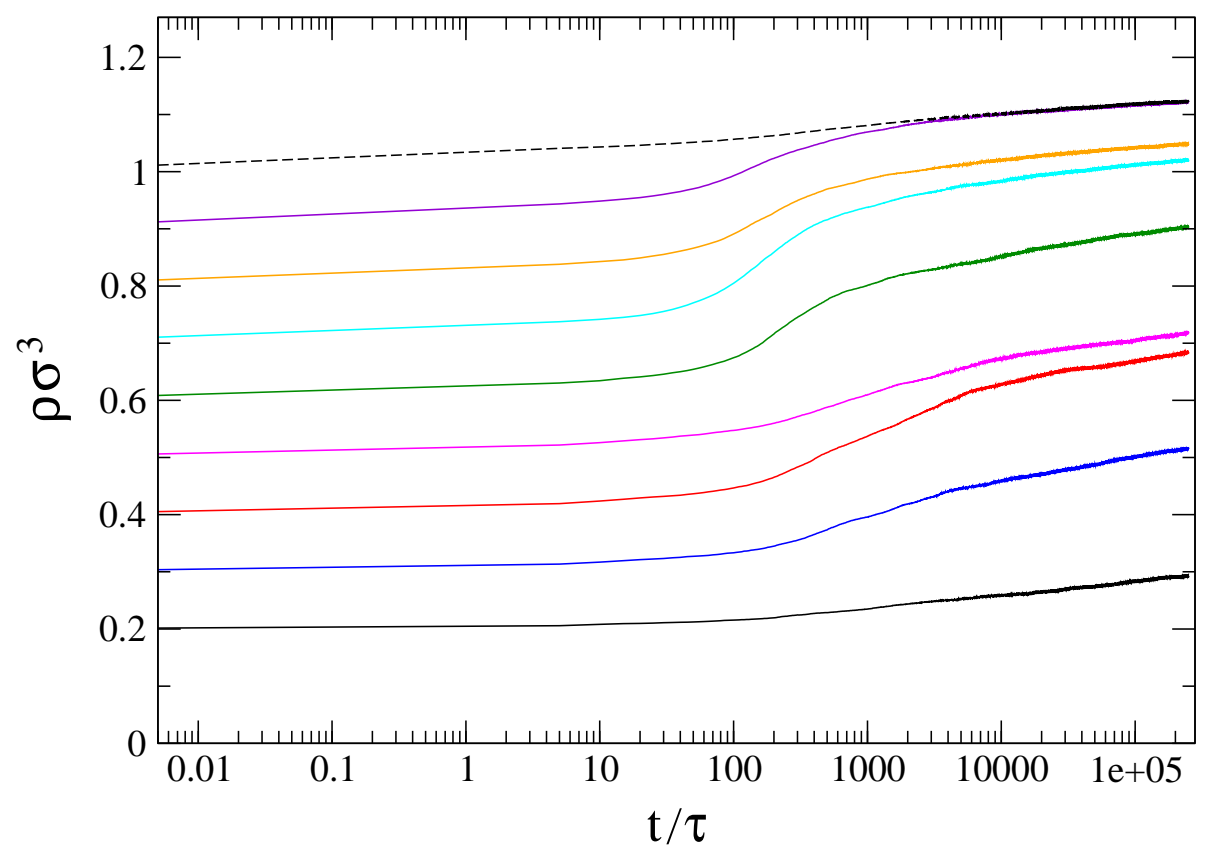

FIG. 2: (Color online) The average glass densities as a function of waiting time during the aging process at zero pressure and temperature $T=0.05 \varepsilon / k_{B}$. The average density of porous samples at $t=0$ is $\rho \sigma^{3}=0.2,0.3,0.4,0.5,0.6,0.7,0.8,0.9$, and 1.0 (from bottom to top). 


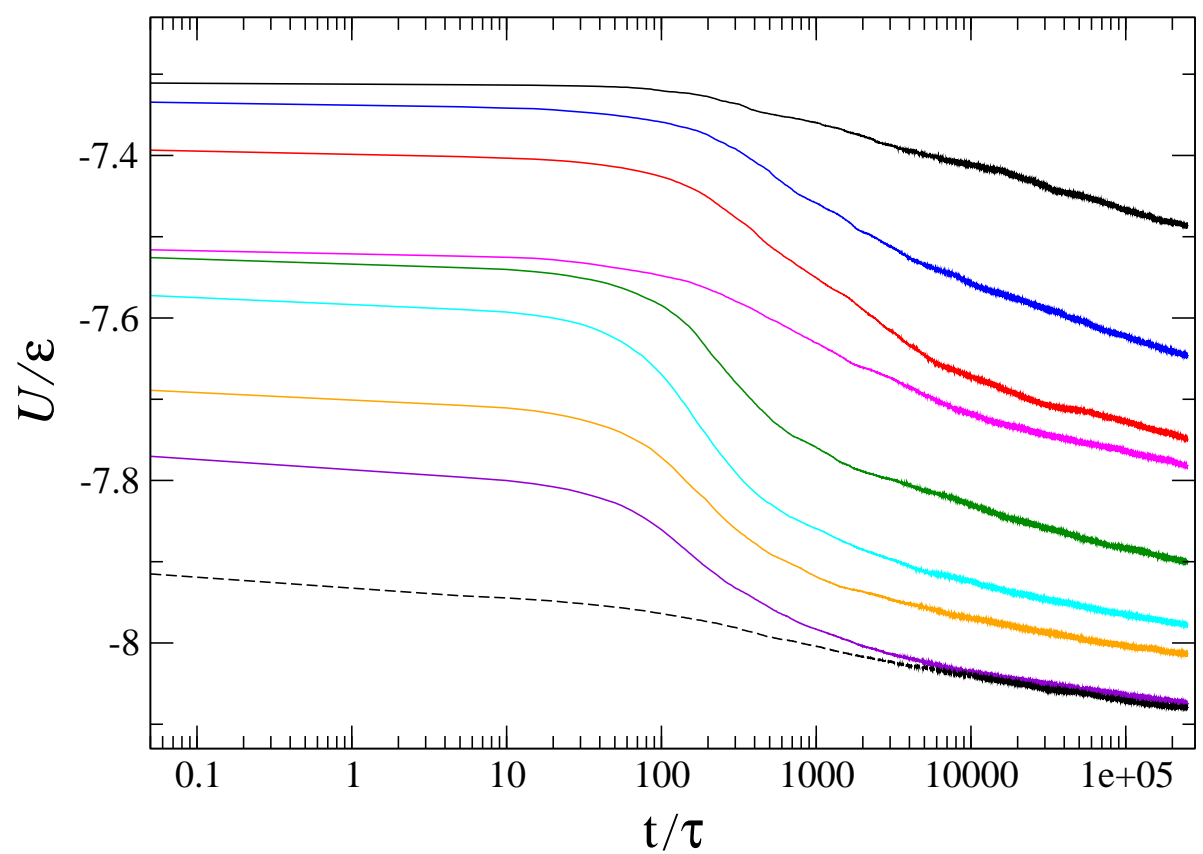

FIG. 3: (Color online) The time dependence of the potential energy per atom for nine samples at constant pressure $P=0$ and $T=0.05 \varepsilon / k_{B}$. The average glass density increases from top to bottom. The same samples and color codes as in Fig.2. 


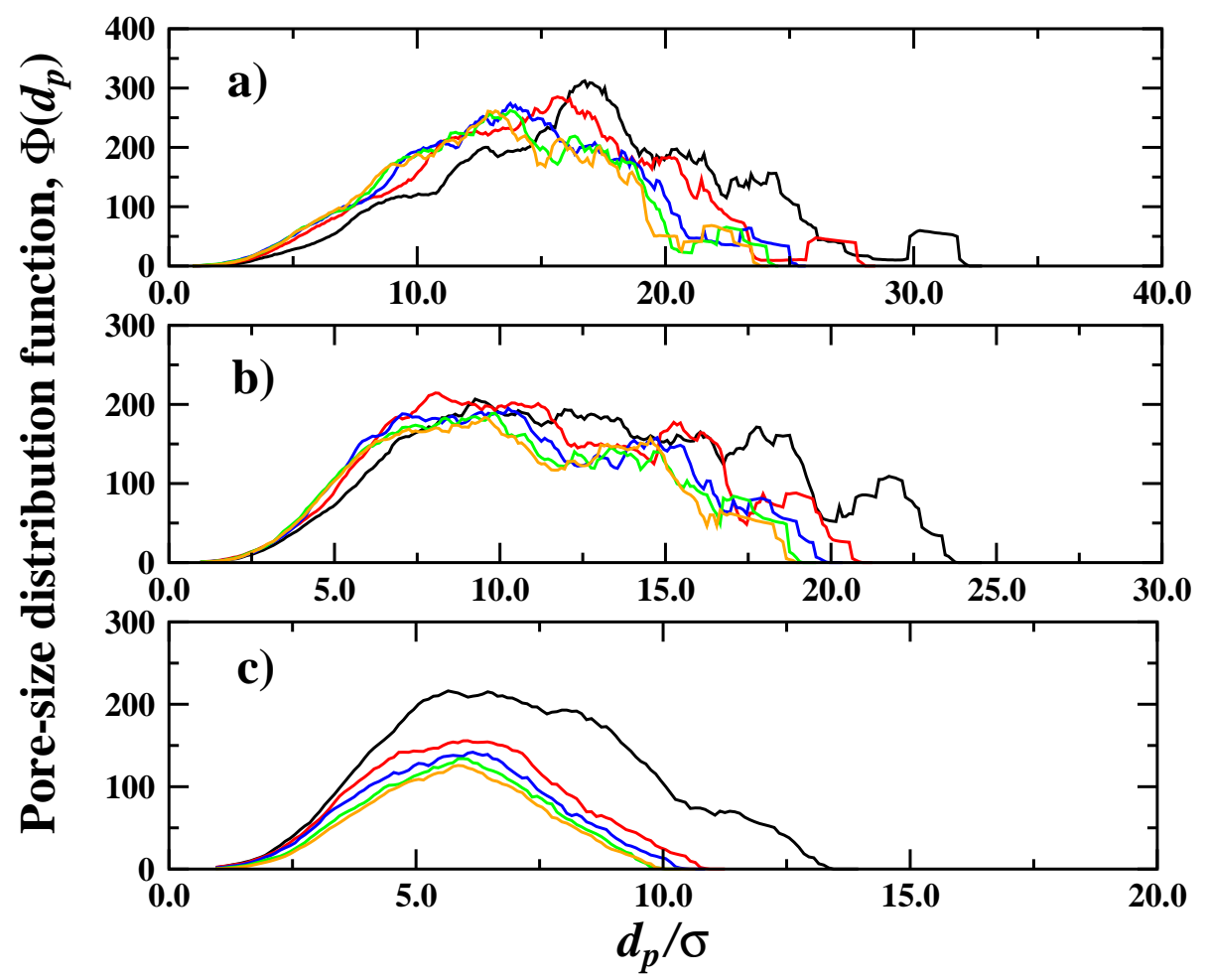

FIG. 4: (Color online) The pore size distributions in quiescent samples during the structural relaxation process at constant pressure. The average glass densities at $t=0$ are (a) $\rho \sigma^{3}=0.3$, (b) $\rho \sigma^{3}=0.5$, and (c) $\rho \sigma^{3}=0.8$. The distribution functions for different waiting times are indicated by black curves $(t=0)$, red curves $\left(t=5 \times 10^{2} \tau\right)$, blue curves $\left(t=5 \times 10^{3} \tau\right)$, green curves $\left(t=5 \times 10^{4} \tau\right)$, and orange curves $\left(t=2.5 \times 10^{5} \tau\right)$. Note that scales are different in three panels. 


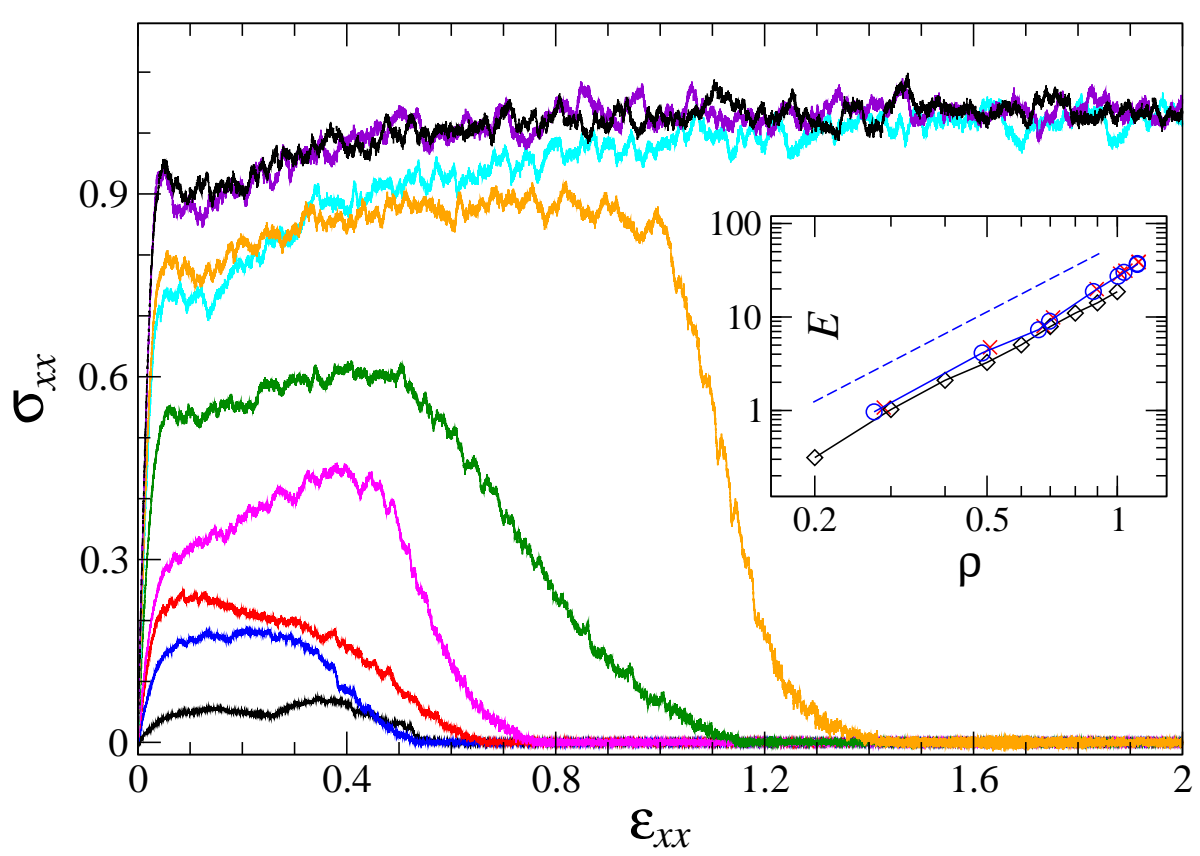

FIG. 5: (Color online) The variation of stress $\sigma_{x x}$ (in units of $\varepsilon \sigma^{-3}$ ) as a function of strain during tensile loading at zero pressure with the strain rate $\dot{\varepsilon}_{x x}=10^{-4} \tau^{-1}$. The samples with different average glass densities were strained after the waiting time of $5 \times 10^{4} \tau$ (see text for details). The inset shows the elastic modulus $E$ (in units of $\varepsilon \sigma^{-3}$ ) as a function of the average glass density $\rho \sigma^{3}$. The data in the inset were computed after the waiting time of $5 \times 10^{4} \tau$ (blue circles) and after $15 \times 10^{4} \tau$ (red crosses). For reference, the data for the elastic modulus at constant volume (taken from Ref. [14]) are also shown by black diamonds. The straight dashed line indicates the slope of 2.41 . 
(a)
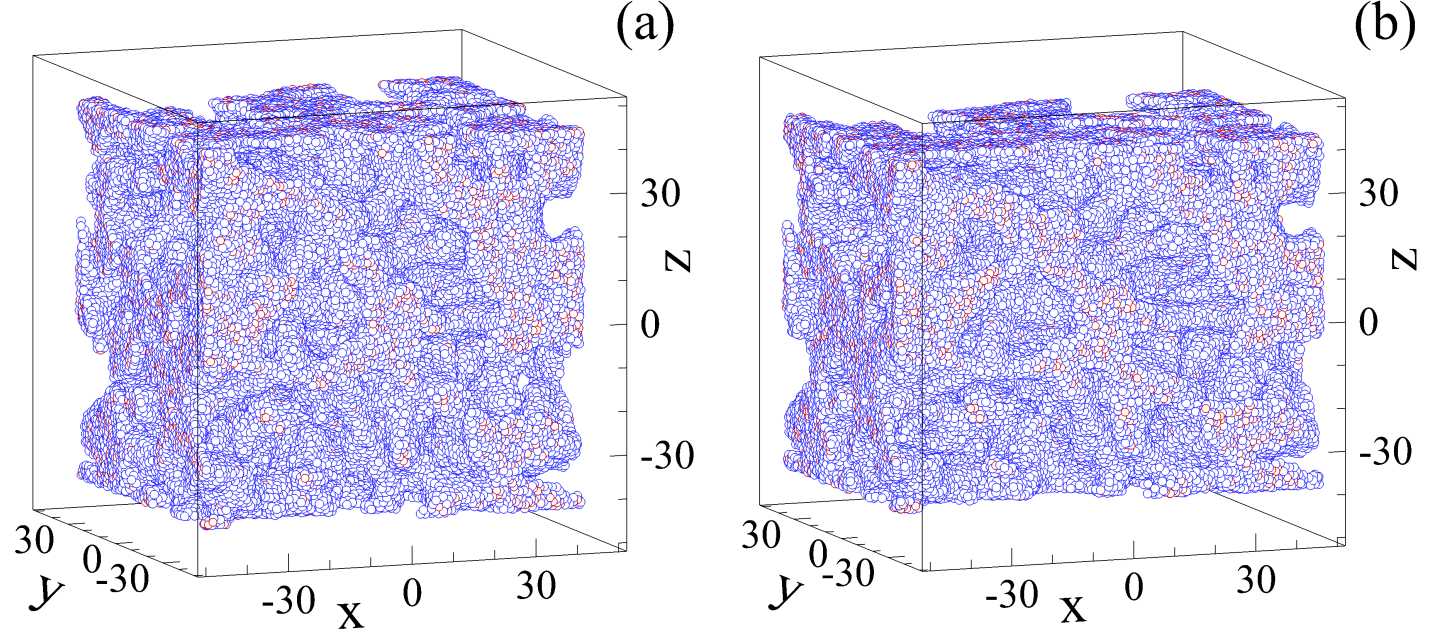

(c)

(d)
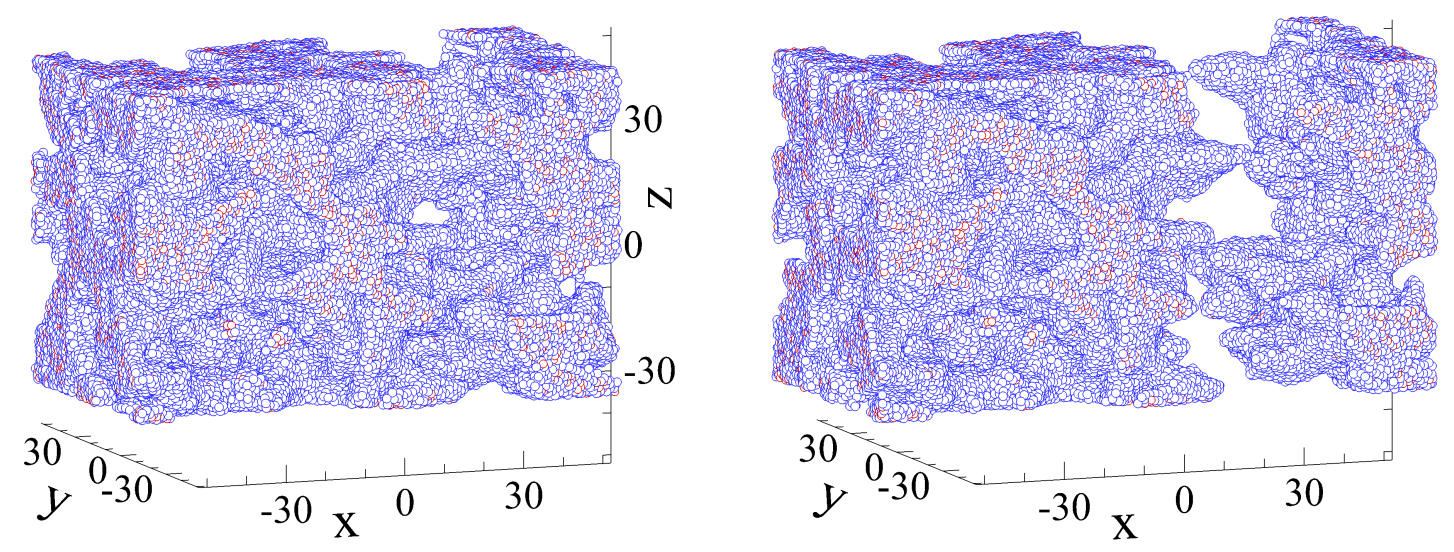

FIG. 6: (Color online) Atomic configurations of the strained porous glass at zero pressure and temperature $T=0.05 \varepsilon / k_{B}$. The values of strain and the average glass density are (a) $\varepsilon_{x x}=0.05$ and $\rho \sigma^{3}=0.48$, (b) $\varepsilon_{x x}=0.20$ and $\rho \sigma^{3}=0.49$, (c) $\varepsilon_{x x}=0.40$ and $\rho \sigma^{3}=0.47$, and (d) $\varepsilon_{x x}=0.60$ and $\rho \sigma^{3}=0.41$. The strain rate is $\dot{\varepsilon}_{x x}=10^{-4} \tau^{-1}$. The stress-strain curve for this sample is marked by blue color in Fig. 5 . 
(a)
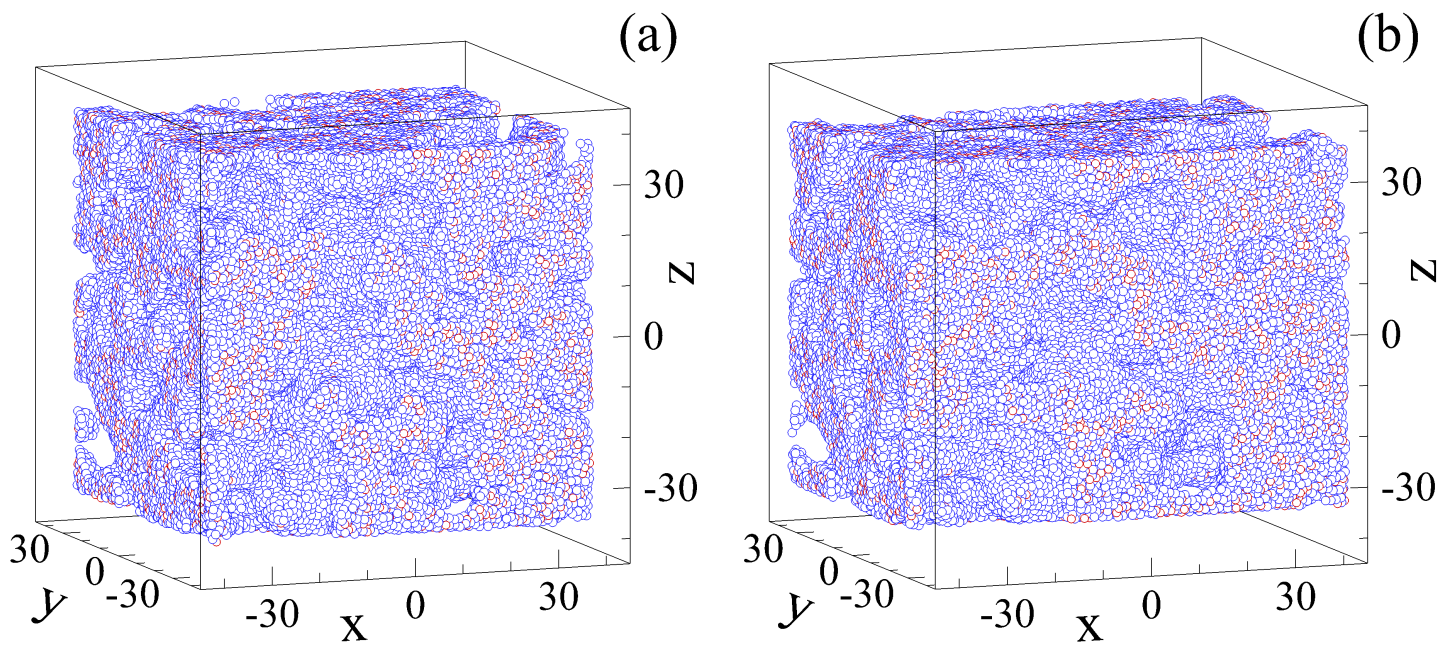

(c)

(d)
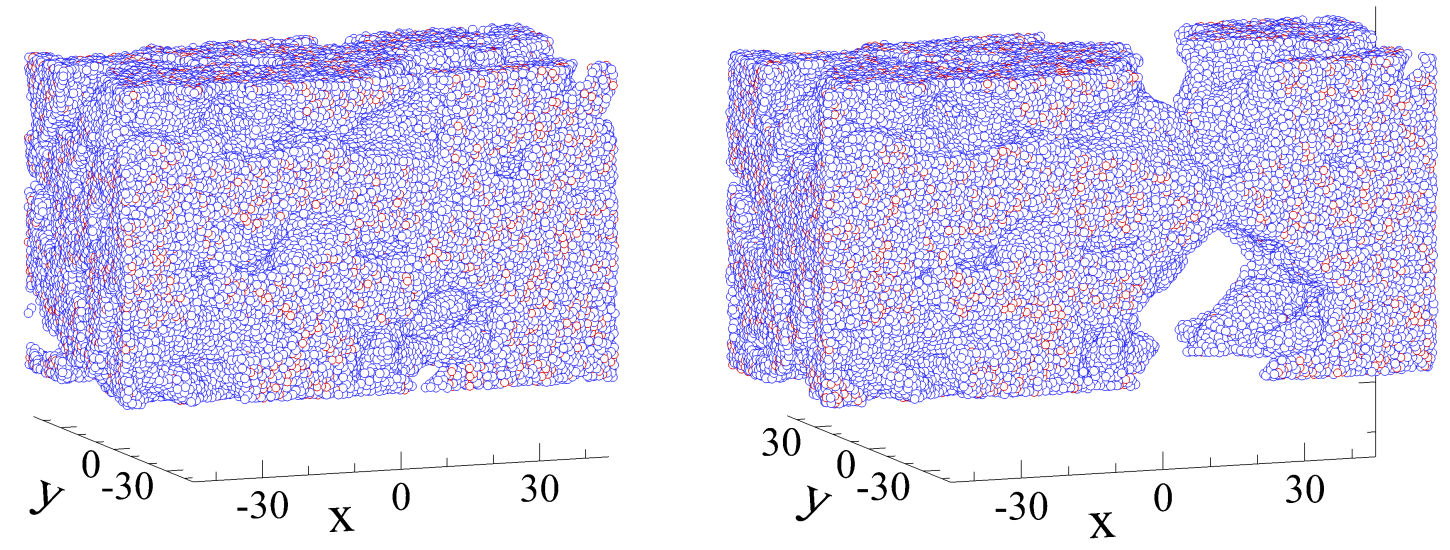

FIG. 7: (Color online) Instantaneous snapshots for the values of strain and the average glass density (a) $\varepsilon_{x x}=0.05$ and $\rho \sigma^{3}=0.69$, (b) $\varepsilon_{x x}=0.20$ and $\rho \sigma^{3}=0.73$, (c) $\varepsilon_{x x}=0.40$ and $\rho \sigma^{3}=0.80$, and (d) $\varepsilon_{x x}=0.70$ and $\rho \sigma^{3}=0.70$. The stress is denoted by the magenta curve in Fig. 5 . 
(a)
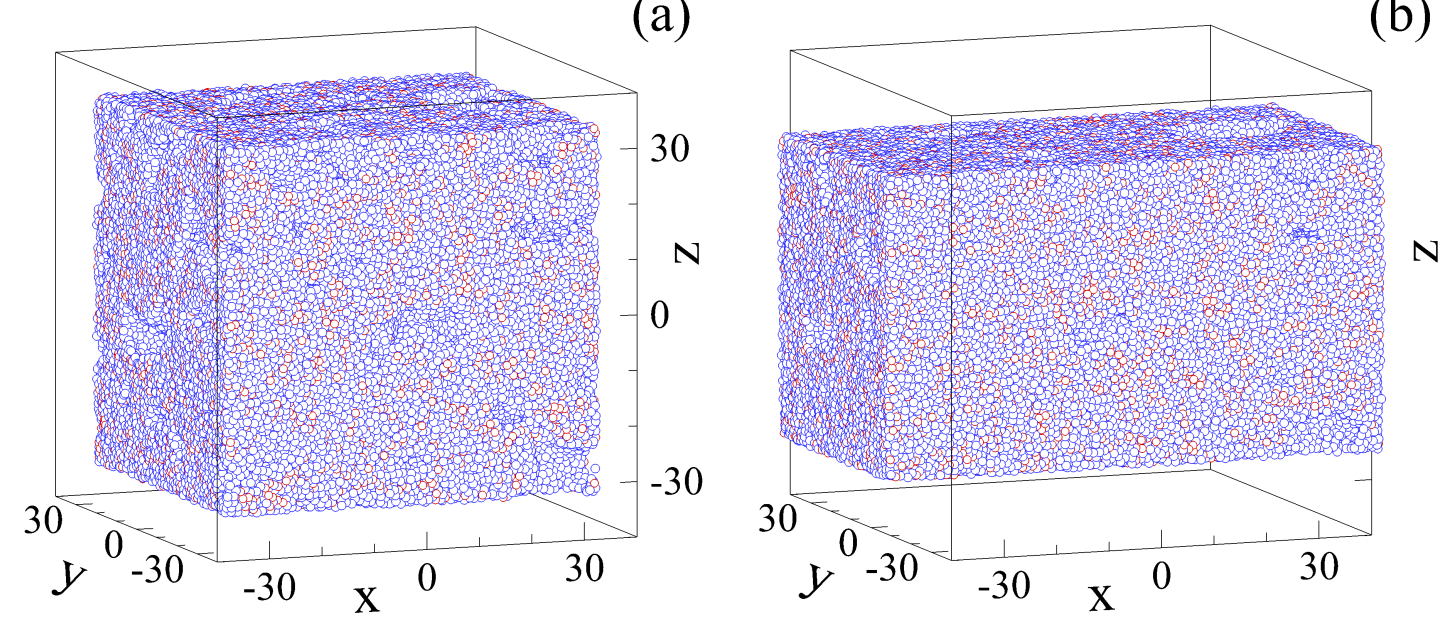

(c)

(d)
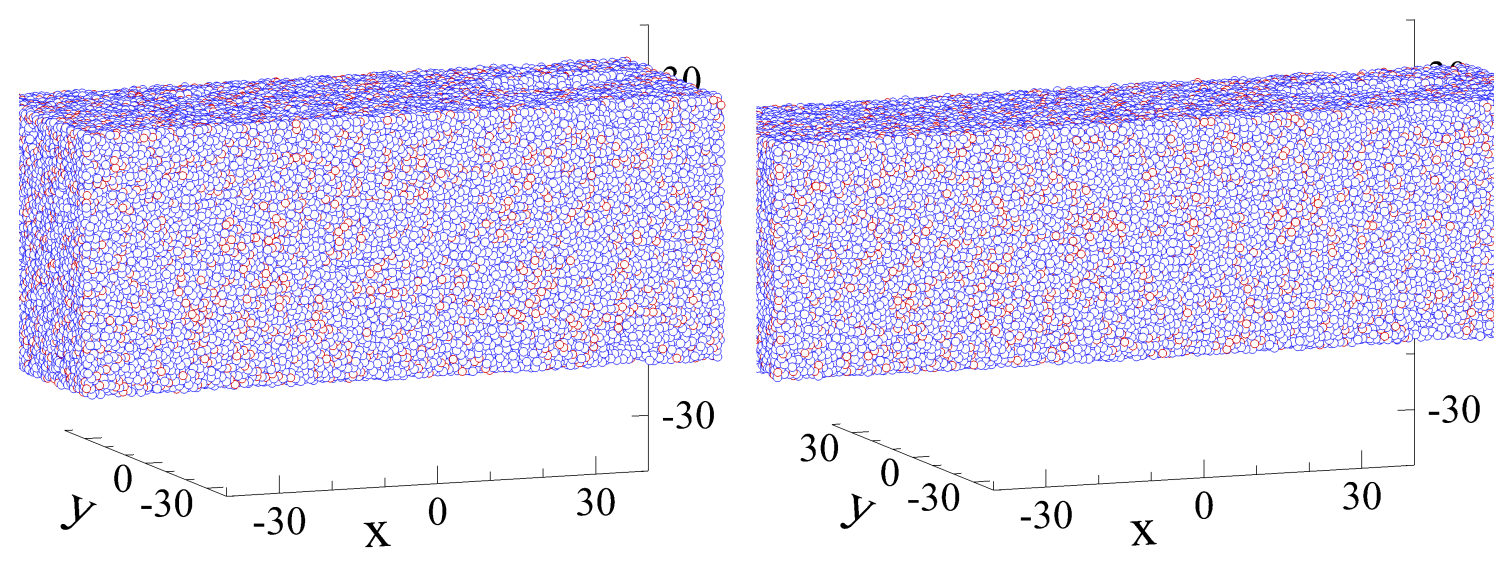

FIG. 8: (Color online) Selected snapshots of the porous glass for strains and average glass densities (a) $\varepsilon_{x x}=0.05$ and $\rho \sigma^{3}=1.0$, (b) $\varepsilon_{x x}=0.40$ and $\rho \sigma^{3}=1.11$, (c) $\varepsilon_{x x}=0.80$ and $\rho \sigma^{3}=1.16$, and (d) $\varepsilon_{x x}=1.60$ and $\rho \sigma^{3}=1.18$. The stress dependence on strain is indicated by the cyan curve in Fig. 5 . 


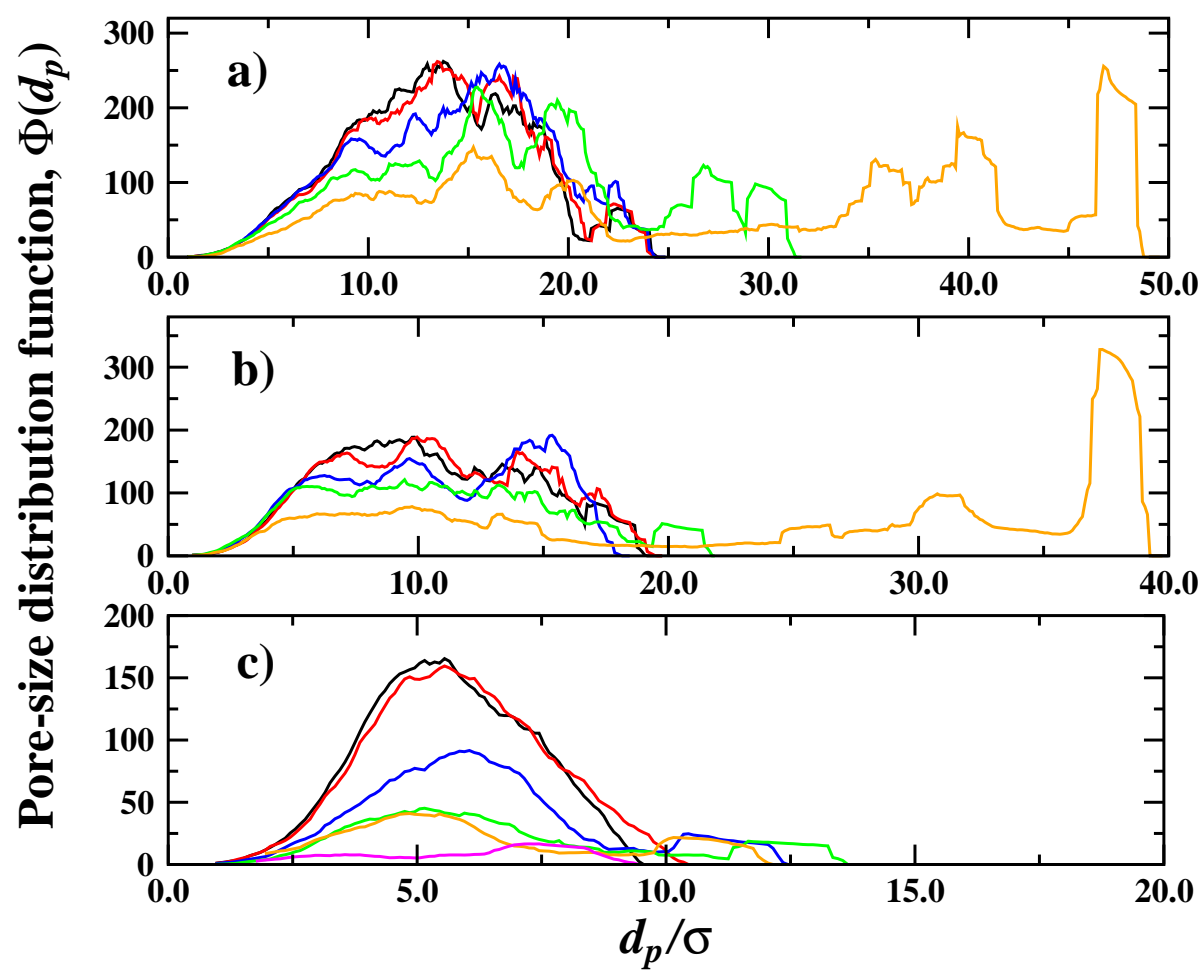

FIG. 9: (Color online) The pore size distribution functions for three samples strained after the waiting time of $5 \times 10^{4} \tau$. The colorcode for different curves is black $\left(\varepsilon_{x x}=0.0\right)$, red $\left(\varepsilon_{x x}=0.05\right)$, blue $\left(\varepsilon_{x x}=0.20\right)$, green $\left(\varepsilon_{x x}=0.40\right)$, orange $\left(\varepsilon_{x x}=0.80\right)$, and magenta $\left(\varepsilon_{x x}=1.60\right)$. The atomic configurations shown in Figs. 6, 7, and 8 are described by the pore size distributions presented in the panels (a), (b), and (c), respectively. 


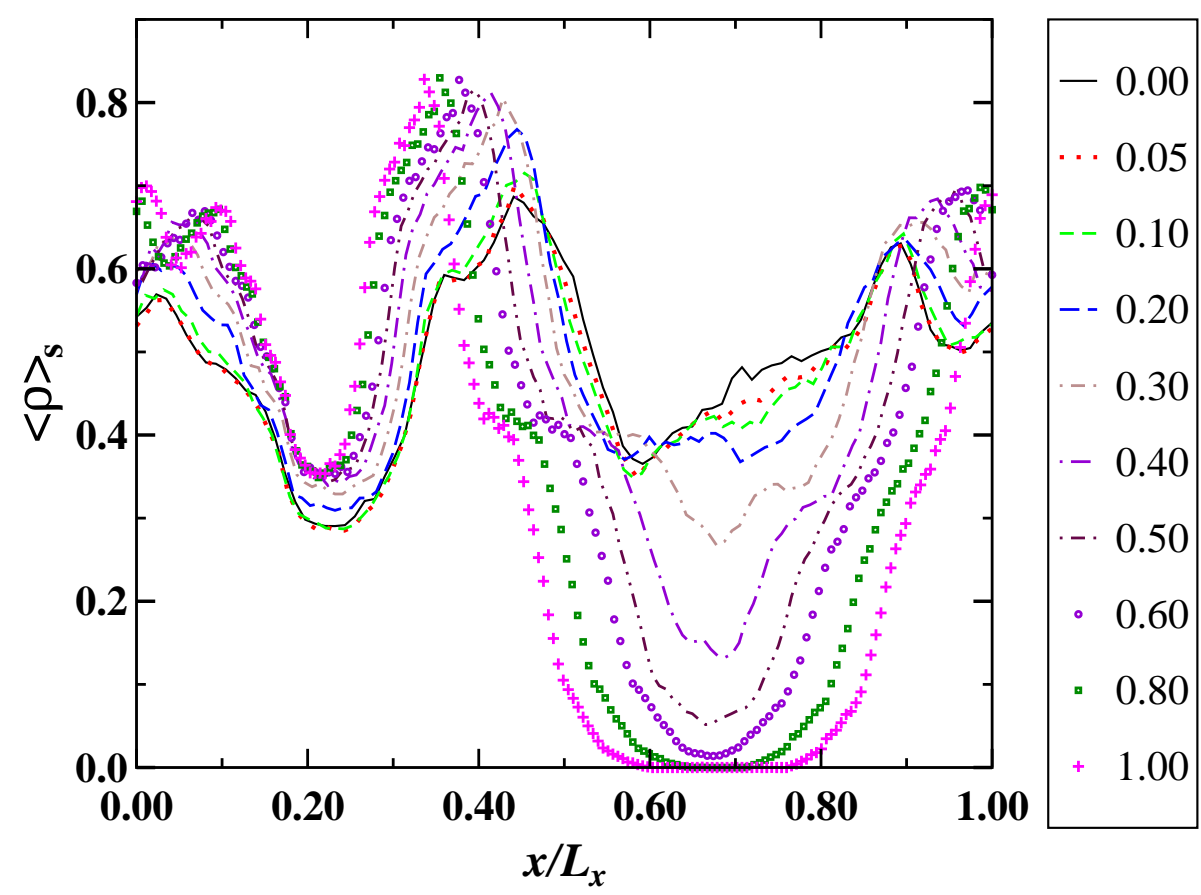

FIG. 10: (Color online) The averaged density profiles $\langle\rho\rangle_{s}(x)$ (in units of $\sigma^{-3}$ ) computed along the $x$-axis (corresponding to the loading direction), are plotted for different values of the applied strain, as shown in the figure legend. The system snapshots are presented in Fig.6 for four values of strain. 


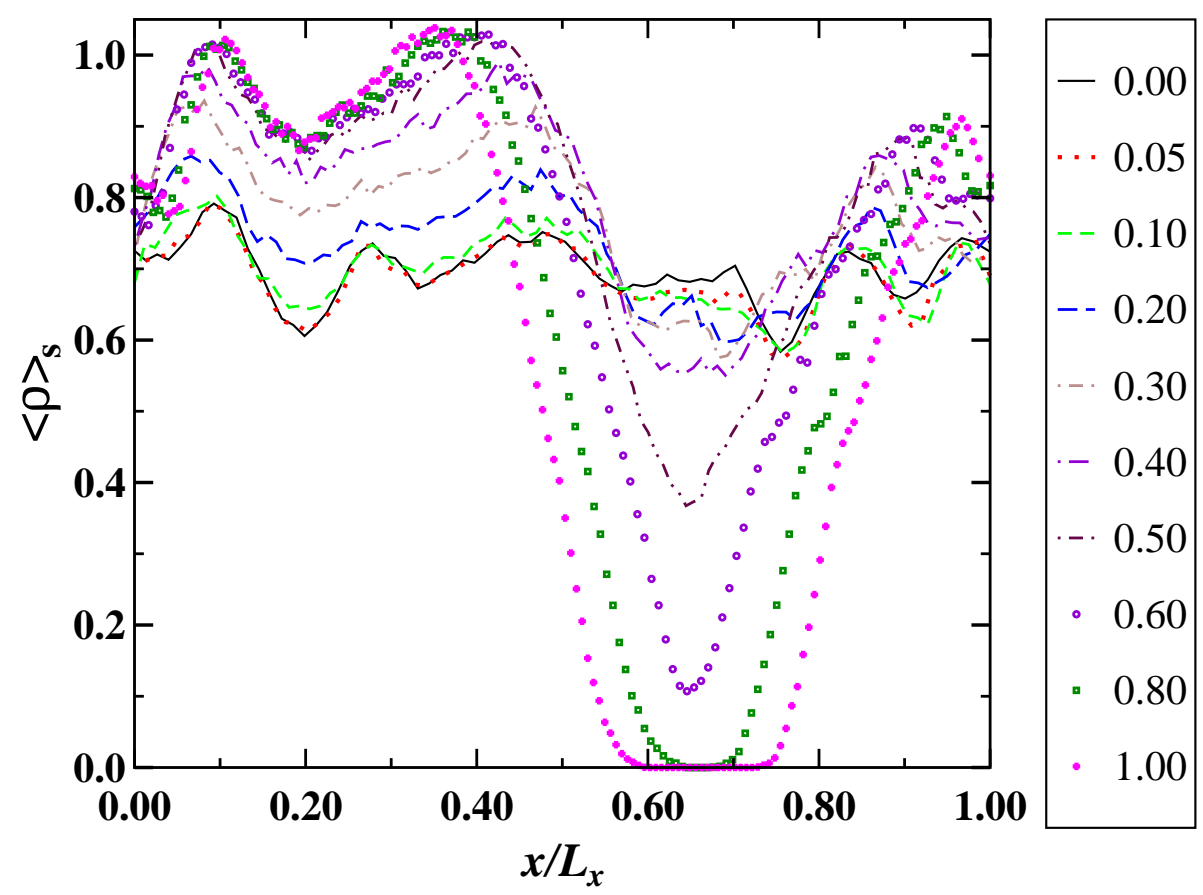

FIG. 11: (Color online) The local density profiles $\langle\rho\rangle_{s}(x)$ (in units of $\sigma^{-3}$ ) along the loading direction are presented for the indicated values of strain. Atomic configurations for this sample are shown in Fig.7 for selected values of strain. 


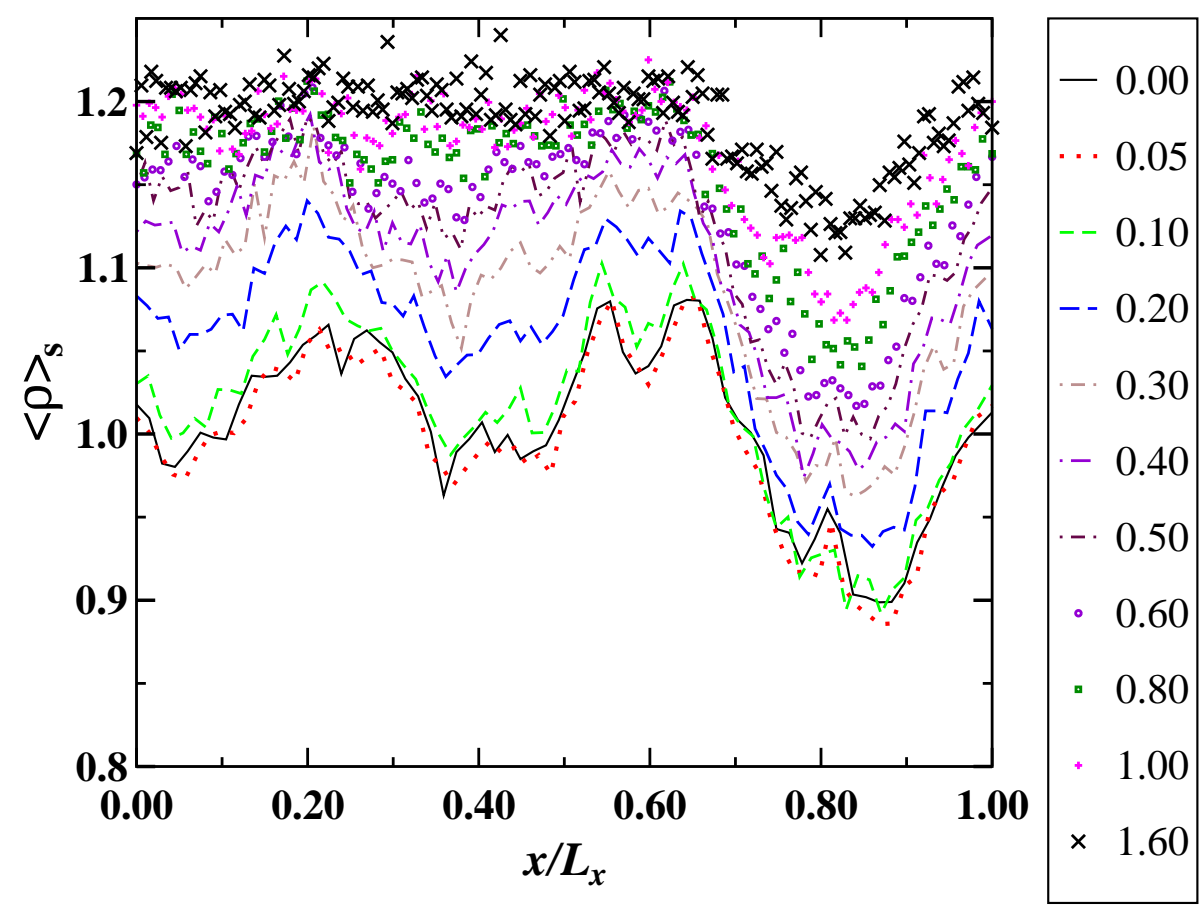

FIG. 12: (Color online) The density profiles $\langle\rho\rangle_{s}(x)$ (in units of $\sigma^{-3}$ ) are plotted for the values of strain listed in the legend. The corresponding atomic configurations are displayed in Fig.8 for selected values of strain. 